IJMMS 31:10 (2002) 619-626

PII. S0161171202112348

http://ijmms.hindawi.com

(c) Hindawi Publishing Corp.

\title{
SOME TYPE OF ROTATIONAL SCHEME
}

\author{
NASHAT FARIED and ZEINAB ABD EL-KADER
}

Received 10 January 2000 and in revised form 27 December 2001

The aim of this paper is to construct a rotational product scheme in the product of Banach spaces with rotational schemes. Besides we give an equivalent definition of the generalized Kolmogorov diameters suggested by Aksoy and Nakamura in 1986, and give the generalized Kolmogorov diameters of the product $\left(\prod_{i \in \mathbb{N}} D_{i}\right.$ ) of bounded subsets $D_{i}$ in the product of Banach spaces having schemes in terms of the generalized diameters of $D_{i}$.

2000 Mathematics Subject Classification: 47Bxx.

1. Introduction. In [3], Pietsch has introduced the concept of an approximation scheme on Banach spaces. In [1], Aksoy has generalized this notion and used it to generalize the concept of Kolmogorov diameters in Banach spaces and in [2] in $p$ Banach spaces.

In this paper, we suggest a method for constructing a product scheme in the product of Banach spaces with schemes. Besides we give an equivalent definition of the generalized Kolmogorov diameters suggested by Aksoy and Nakamura in [2] and give the generalized Kolmogorov diameters of the product $\left(\prod_{i \in \mathbb{N}} D_{i}\right)$ of bounded subsets $D_{i}$ in the product of Banach spaces having schemes in terms of the generalized diameters of $D_{i}$.

In terms of the equivalent definition of the generalized Kolmogorov diameters, we study analogous result for a sequence of generalized Kolmogorov diameters of bounded linear operators.

2. Notations and definitions. We denote by $L(E, X)$ and $\mathscr{F}_{n}(E, X)$ the normed spaces of bounded and at most $n$-dimensional linear operators from the normed space $E$ into the normed space $X$.

For a sequence $\left\{X_{i}\right\}_{i \in \mathbb{N}}$ of Banach spaces, and for any $k \in \mathbb{N}$, we denote by $\ell_{\infty}\left(X_{i}\right)$ and $\ell_{p}^{k}\left(X_{i}\right)$ the Banach spaces

$$
\begin{aligned}
& \ell_{\infty}\left(X_{i}\right)=\left\{x=\left(x_{i}\right)_{i \in \mathbb{N}}: x_{i} \in X_{i}, \sup _{i \in \mathbb{N}}\left\|x_{i}\right\|_{X_{i}}<\infty\right\}, \\
& \ell_{p}^{k}\left(X_{i}\right)=\left\{x=\left(x_{i}\right)_{i \in \mathbb{N}}: x_{i} \in X_{i}, 1 \leq i \leq k\right\}
\end{aligned}
$$

equipped with the norms

$$
\|x\|=\sup _{i \in \mathbb{N}}\left\|x_{i}\right\|_{X_{i}}, \quad\|x\|=\left(\sum_{i=1}^{k}\left\|x_{i}\right\|_{X_{i}}^{p}\right)^{1 / p},
$$

respectively. 
For a natural number $n \in \mathbb{N}$, we denote by $\beta_{n}$ a function from the set $\mathbb{N}$ of natural numbers into the set $\mathbb{N}^{*}=\mathbb{N} \cup\{0\}$ for which $\sum \beta_{n}(i)=n_{i}$, it is true that $\beta_{n}(i)=0$ for all $i \in \mathbb{N}$ except a finite number, we write $\beta_{n}(i)=n_{i}$.

By $d(x, F)=\inf _{y \in F}\|x-y\|$ we denote the distance between an element $x \in X$ and a subset $F$ of a Banach space $X$.

For the families $\mathscr{E}_{i}$ of subsets of the Banach spaces $X_{i}$, we denote by $\prod_{i \in \mathbb{N}} \mathscr{E}_{i}$ the product family $\left\{\prod_{i \in \mathbb{N}} A_{i}: A_{i} \in \mathscr{E}_{i}\right\}$ in the product space $\prod_{i \in \mathbb{N}} X_{i}$.

DEFINITION 2.1 (rotational operators). An operator $\mathscr{R}$ from a normed space $X$ into itself is called a rotational operator if and only if it can be written as $\mathscr{R}=R_{1} R_{2} \cdots R_{k}$ with the following conditions:

(1) $\inf _{n \in \mathbb{N}}\left\|R_{i}^{n}-I\right\|=0, i=1,2, \ldots, k$,

(2) $\left\|R_{i}\right\|=\left\|R_{i}^{-1}\right\|=1, i=1,2, \ldots, k$,

where $I$ is the identity operator from $X$ into itself.

REMARK 2.2. In [4], Pietsch has introduced the notion of approximation scheme (satisfying conditions $\left(\mathrm{S}_{1}\right),\left(\mathrm{S}_{2}\right)$, and $\left(\mathrm{S}_{3}\right)$ in Definition 2.3$)$. Here we give a slight modification of that definition and call it a rotational approximation scheme.

DEFINITION 2.3 (rotational approximation scheme). Let $X$ be a normed space over a field of scalars $K$, for each $n \in \mathbb{N}$, let $Q_{n}=Q_{n}(X)$ be a family of subsets of $X$ satisfying the following conditions:

$\left(\mathrm{S}_{1}\right)\{0\}=Q_{0}(X) \subset Q_{1}(X) \subset \cdots \subset Q_{n}(X) \subset \cdots$.

$\left(\mathrm{S}_{2}\right)$ If $A \in Q_{n}(X)$ and $\lambda \in K$, then $\lambda A \in Q_{n}(X)$ for every $n \in \mathbb{N}^{*}$.

$\left(\mathrm{S}_{3}\right)$ If $A \in Q_{n}(X)$ and $B \in Q_{m}(X)$, then $A+B \in Q_{n+m}$ for every $n, m \in \mathbb{N}^{*}$.

$\left(\mathrm{S}_{4}\right)$ If $A \in Q_{n}(X)$ and $\mathscr{R}$ is a rotational operator, then $\mathscr{R} A \in Q_{n}(X)$.

Then $Q(X)=\left\{Q_{n}(X)\right\}_{n \in \mathbb{N} *}$ is called a rotational approximation scheme (invariant under rotation).

EXAMPLE 2.4. In any Banach space $X$, the scheme $\mathscr{F}(X)=\left(\mathscr{F}_{n}(X)\right)_{n \in \mathbb{N}}$ consisting of all finite-dimensional subspaces $\mathscr{F}_{n}(X)$, where

$$
\mathscr{F}_{n}(X)=\{F \subseteq X: \operatorname{dim} F \leq n\},
$$

is a rotational approximation scheme in $X$.

EXAMPLE 2.5. The class $\mathscr{A}=\left\{\mathscr{A}_{n}(L(X))\right\}_{n \in \mathbb{N}}$ of all finite-dimensional operators on a Banach space forms a rotational approximation scheme on the Banach space $L(X)$ of all bounded linear operators on $X$, where

$$
\mathscr{A}_{n}(L(X))=\{T \in L(X): \operatorname{rank} T \leq n\}
$$

We only remark that an operator $T \in \mathscr{A}_{n}(L(X))$ is finite dimensional (of finite rank) if and only if $T$ has as a representation

$$
T x=\sum_{i=1}^{n} f_{i}(x) y_{i} ; \quad f_{i} \in X^{*}, y_{i} \in X
$$


EXAMPLE 2.6. The class of all finite subsets $\tau=\left[\tau_{n}(X)\right]_{n \in \mathbb{N}}$ in any Banach space $X$ forms a rotational approximation scheme, where

$$
\tau_{n}(X)=\left\{A \subseteq X: \operatorname{card} A \leq 2^{n}\right\} .
$$

For $A \in \tau_{n}(X)$ and $B \in \tau_{m}(X)$, we note that

$$
\operatorname{card}(A+B) \leq(\operatorname{card} A)(\operatorname{card} B)=2^{n+m} .
$$

Hence $A+B \in \tau_{n+m}(X)$.

REMARK 2.7. In [4], Pietsch has given a generalized definition for Kolmogorov diameters as follows.

DEFINITION 2.8. Let $X$ be a Banach space with closed unit ball $U \subset X$ and equipped with an approximation scheme $Q(X)$. The $n$th Kolmogorov diameter $\delta_{n}(D)$ of a bounded subset $D$ of $X$ is defined by

$$
\delta_{n}(D)=\inf \left\{\lambda>0: D \subset \lambda U+A \text { for some } A \in Q_{n}(X)\right\} .
$$

In fact, in case $Q_{n}(X)$ is the class of all finite-dimensional subspaces $\mathscr{F}_{n}(X)$, relation (2.8) reduces to Kolmogorov diameters mentioned in [3].

REMARK 2.9. In [1], Aksoy has given a slight modification for Definition 2.8 namely, the following definition.

DEFINITION 2.10. Let $X$ be a Banach space with closed unit ball $U \subset X$ and equipped with an approximation scheme $Q(X)$. The $n$th generalized diameter $v_{n}(D)$ of a bounded subset $D$ of $X$ is defined by

$$
\begin{aligned}
& v_{n}(D)=\inf \left\{\lambda>0: D \subset \lambda U_{X}+T U_{E}: T U_{E} \subset A\right. \\
& \text { for some Banach space } \left.E \text { and some } A \in Q_{n}(X)\right\} .
\end{aligned}
$$

From now on, we are going to deal with the following notations to simplify our definitions and proofs: let $X$ be a Banach space with a rotational approximation scheme $Q=\left(Q_{n}(X)\right)_{n \in \mathbb{N}^{*}}$, and let $U_{E}$ be the unit ball of some Banach space $E$. We write

$$
\begin{aligned}
\mathscr{L}\left(Q_{n}(X), E, X\right)= & \left\{T \in L(E, X), T\left(U_{E}\right) \subset A \text { for some } A \in Q_{n}(X)\right\}, \\
& \mathscr{L}\left(Q_{n}(X)\right)=\bigcup_{E \in X} \mathscr{L}\left(Q_{n}(X), E, X\right),
\end{aligned}
$$

where $\chi$ is the category of all Banach spaces.

REMARK 2.11. Using these notations we can write the space of all finite-dimensional operators from $E$ to $X$ as $\mathscr{L}\left(\mathscr{F}_{n}(X), E, X\right)$, and $\mathscr{L}\left(\mathscr{F}_{n}(X)\right)$ as the collection of all finitedimensional operators with range in $X$, that is, $\mathscr{L}\left(\mathscr{F}_{n}(X), E, X\right)=\mathscr{F}_{n}(X, E)$. 
Definition 2.10 can be written in terms of the previous notations as follows:

$$
\nu_{n}(D)=\inf \left\{\lambda>0: D \subset \lambda U_{X}+T\left(U_{E}\right) \text { and } T \in \mathscr{L}\left(Q_{n}(X)\right)\right\} .
$$

In the following lemma, we give an equivalent definition for $v_{n}(D)$ as follows.

LEMMA 2.12. For a bounded subset $D$ of a Banach space $X$, it is true that

$$
v_{n}(D)=\inf _{T \in \mathscr{L}\left(Q_{n}(X)\right)} \sup _{x \in D} d\left(x, T U_{E}\right) .
$$

Proof. Let $\sigma_{n}(D)=\inf _{T \in \mathscr{L}\left(Q_{n}(X)\right)} \sup _{x \in D} d\left(x, T U_{E}\right)$. From the definition of $v_{n}(D)$ and for every $\epsilon>0$, there exists $\lambda>0$ such that

$$
v_{n}(D) \leq \lambda<v_{n}(D)+\epsilon
$$

with

$$
D \subset \lambda U_{X}+T\left(U_{E}\right) \subset\left(v_{n}(D)+\epsilon\right) U_{X}+T\left(U_{E}\right) .
$$

Then every element $x \in D$ can be written as

$$
x=\left(v_{n}(D)+\epsilon\right) u_{x}+y_{x} \text { for } u_{x} \in U_{X}, y_{x} \in T\left(U_{E}\right)
$$

Then

$$
d\left(x, T U_{E}\right)=\inf _{y \in T U_{E}}\|x-y\| \leq\left\|x-y_{x}\right\| \leq v_{n}(D)+\epsilon .
$$

Therefore,

$$
\sigma_{n}(D)=\inf _{T \in \mathscr{L}\left(Q_{n}(X)\right)} \sup _{x \in D} d\left(x, T U_{E}\right) \leq \nu_{n}(D)+\epsilon .
$$

From the definition of $\sigma_{n}(D)$ and for any positive number $\epsilon>0$ and any $T \in \mathscr{L}\left(Q_{n}(X)\right)$, we get

$$
d\left(x, T U_{E}\right) \leq \sup _{x \in D} d\left(x, T U_{E}\right)<\sigma_{n}(D)+\epsilon .
$$

Then for $x \in D$ there exists an element $T u \in T U_{E}$ such that

$$
\sigma_{n}(D)>\|x-T u\| .
$$

Since every element $x \in D$ can be written as $x=(x-T u)+T u$, then

$$
D \subset\left(\sigma_{n}(D)+\epsilon\right) U+T_{E},
$$

so

$$
v_{n}(D) \leq \sigma_{n}(D)+\epsilon .
$$

From (2.17) and (2.21), we get the proof. 
DEFINITION 2.13. For an operator $S \in L(E, X)$, the sequence of Kolmogorov numbers $\left\{\zeta_{n}(S)\right\}_{n \in \mathbb{N}}$ is defined by

$$
\zeta_{n}(S)=v_{n}\left(S U_{E}\right)
$$

2.1. Rotational product scheme. We suggest a construction of a rotational approximation scheme in the product of Banach spaces with rotational schemes, using the following definition of a base of a rotational approximation scheme.

2.2. Base of a rotational approximation scheme. In the Banach space $\mathscr{R}^{1}$, the scheme of finite-dimensional subspaces consists only of the two trivial subspaces $\{0\}, \mathscr{R}$. However in $\mathscr{R}^{2}$, the finite-dimensional subspaces are not only the four basic subspaces $\{0\} \times\{0\},\{0\} \times \mathscr{R}, \mathscr{R} \times\{0\}, \mathscr{R} \times \mathscr{R}$, but contains also all their rotations, for example, $L=\{(x, x): x \in \mathscr{R}\}$ is a finite-dimensional subspace; $L=I_{\pi / 4}(\mathscr{R} \times 0)$ where

$$
I_{\pi / 4}=\left(\begin{array}{cc}
\frac{1}{\sqrt{2}} & -\frac{1}{\sqrt{2}} \\
\frac{1}{\sqrt{2}} & \frac{1}{\sqrt{2}}
\end{array}\right)=\left(\begin{array}{cc}
\cos \frac{\pi}{4} & -\sin \frac{\pi}{4} \\
\sin \frac{\pi}{4} & \cos \frac{\pi}{4}
\end{array}\right) .
$$

So we conclude from this example that, in general, to construct an approximation scheme in the product $X \times Y$ of two Banach spaces with schemes $(X, Q(X))$ and $(Y, Q(Y))$, it is not sufficient to take for $Q_{n}(X \times Y)$ the set

$$
\left\{A \times B: A \in Q_{i}(X), B \in Q_{n-i}(Y), 0 \leq i \leq n\right\} .
$$

In fact, we will also consider all their rotations. This motivated the following definition.

DefinITION 2.14. A base $Q_{0}(X)=\left(Q_{n}^{o}(X)\right)_{n \in \mathbb{N}^{*}}$ of a rotational scheme $Q(X)$ is a subcollection of $Q(X)$ satisfying the following conditions:

(1) $Q_{n}^{o}(X) \subseteq Q_{n}(X)$ for every $n \in \mathbb{N}^{*}$.

(2) For every $A \in Q_{n}(X)$, there exist a rotational operator $R_{A}$ and an element $A_{o} \in$ $Q_{n}^{o}(X)$ such that $A=R_{A} A_{o}$.

In $\mathscr{R}^{2}$ the approximation scheme of finite-dimensional subspaces $F\left(\mathscr{R}^{2}\right)$ has a base

$$
Q^{o}\left(\mathscr{R}^{2}\right)=\{\{0\} \times\{0\},\{0\} \times \mathscr{R}, \mathscr{R} \times\{0\}, \mathscr{R} \times \mathscr{R}\} .
$$

All other subspaces of $\mathscr{R}^{2}$ can be obtained by rotations of subspaces in the base. For example, $L=\{(x, x): x \in \mathscr{R}\}=I_{\pi / 4} \mathscr{R}$.

Definition 2.15 (rotational product scheme). Let $\left(X_{i}\right)_{i \in \mathbb{N}}$ be a sequence of Banach spaces each of them equipped with a rotational approximation schemes $Q\left(X_{i}\right)=$ $\left\{Q_{n}\left(X_{i}\right)\right\}_{n \in \mathbb{N} *}$ for every $i \in \mathbb{N}$.

Now to construct the product scheme $Q(X)$ in the product space $\prod X_{i}$ we first choose $Q_{n}^{o}(X)$ as follows:

$$
Q_{n}^{o}(X)=\left\{c: c=\prod_{i \in \mathbb{N}} A_{i}: A_{i} \in Q_{n_{i}}\left(X_{i}\right): \sum n_{i} \leq n\right\} .
$$


We take $\left(Q_{n}^{o}(X)\right)_{n \in \mathbb{N}}$ to be a basis for a rotational scheme namely, $Q(X)=\left(Q_{n}(X)\right)_{n \in \mathbb{N}}$ where

$$
Q_{n}(X)=\left\{B: B=R C, C \in Q_{n}^{o}(X), R \in \mathscr{R}(X)\right\}
$$

and $\mathscr{R}(X)$ is the space of all rotational operators $R \in L(X)$.

2.3. Verification of rotational approximation scheme axioms. In fact,

$\left(\mathrm{S}_{1}\right) Q_{o}(X)=\left\{B: B=\mathscr{R} C, C=\prod\{0\}:\{0\} \in Q_{o}\left(X_{i}\right)\right\}=\{0\} \subset Q_{1}(X) \subset \cdots$.

$\left(\mathrm{S}_{2}\right)$ Let $B \in Q_{n}(X)$ and $\lambda \in K$ then $B=R C$ for some $C=\prod_{i \in \mathbb{N}} A_{i}$, with $A_{i} \in Q_{n_{i}}\left(X_{i}\right)$, $\sum n_{i} \leq n$. Since $\lambda C \in Q_{n}^{o}(X)$, then $\lambda B=\lambda R C=R(\lambda C) \in Q_{n}(X)$.

$\left(\mathrm{S}_{3}\right)$ If $A \in Q_{n}(X), B \in Q_{m}(X)$, then there exist rotational operators $R^{1}, R^{2} \in L(X)$ and $A^{o}=\prod A_{i} \in Q_{n}^{o}(X), B^{o}=\prod B_{i} \in Q_{m}^{o}(X)$ with $A_{i} \in Q_{n_{i}}\left(X_{i}\right), \sum n_{i} \leq n$ and $B_{i} \in$ $Q_{m_{i}}\left(X_{i}\right), \sum m_{i} \leq m$ such that $A=R^{1} A^{o}, B=R^{2} B^{o}$. Therefore,

$$
A+B=R^{1}\left(\prod A_{i}\right)+R^{2}\left(\prod B_{i}\right)
$$

Therefore,

$$
A+B \in Q_{n+m}(X) .
$$

$\left(\mathrm{S}_{4}\right)$ If $A \in Q_{n}(X)$ then $A=R C=R \prod_{i \in \mathbb{N}} A_{i}$ and if $S$ is a rotational operator, then $S A=S R C=T C$, where $T$ is a rotational operator in $L(X)$, then $S A \in Q_{n}(X)$.

We need the following lemma.

LEMMA 2.16. Let $\left\{X_{i}\right\}_{i \in \mathbb{N}}$ be a sequence of Banach spaces, with closed unit balls $\left\{U_{i}\right\}_{i \in \mathbb{N}}$. The unit balls of the Banach spaces $\ell_{\infty}\left(X_{i}\right)$ and $\ell_{p}^{k}\left(X_{i}\right)$ satisfy the following conditions:

(1) $U_{\ell_{\infty}(X)}=\ell_{\infty}\left(U_{i}\right)$;

(2) $\left(1 / k^{1 / p}\right) \ell_{p}^{k}\left(U_{i}\right) \subset U_{\ell_{p}^{k}\left(X_{i}\right)} \subset \ell_{p}^{k}\left(U_{i}\right)$.

The proof follows by simple verifications.

THEOREM 2.17. For the Banach spaces $\ell_{\infty}\left(X_{i}\right)$ and $\ell_{p}^{k}\left(X_{i}\right)$,

(1) $\mathscr{L}\left(Q_{n}\left(\ell_{\infty}\left(X_{i}\right)\right)\right)=\bigcup_{\sum n_{i} \leq n} \prod_{i \in \mathbb{N}} \mathscr{L}\left(Q_{n_{i}}\left(X_{i}\right)\right)$;

(2) $\mathscr{L}\left(Q_{n}\left(\ell_{p}^{k}\left(X_{i}\right)\right)\right) \supset\left(1 / k^{1 / p}\right) \bigcup_{\sum n_{i} \leq n} \prod_{i \in \mathbb{N}} \mathscr{L}\left(Q_{n_{i}}\left(X_{i}\right)\right)$.

Proof. From Remark 2.2 and Lemma 2.16 we get

$$
\begin{aligned}
& \mathscr{L}\left(Q_{n}\left(\ell_{\infty}\left(X_{i}\right)\right)\right)=\left\{\prod_{i \in \mathbb{N}} T_{i}:\left(\prod_{i \in \mathbb{N}} T_{i}\right)\left(U_{\ell_{\infty}\left(E_{i}\right)_{i \in \mathbb{N}}}\right) \subset A_{n}\right\} \\
& \text { for some } A_{n} \in Q_{n}\left(\ell_{\infty}\left(X_{i}\right)_{i \in \mathbb{N}}\right) \\
&= \prod_{i \in \mathbb{N}}\left\{T_{i}: T_{i} \in \mathscr{L}\left(Q_{n_{i}}\left(X_{i}\right), E_{i}, X_{i}\right), T_{i}\left(U_{E_{i}}\right) \subset A_{n_{i}}\right\} \\
& \quad \text { for some } A_{n_{i}} \in Q_{n_{i}}\left(X_{i}\right), \sum n_{i} \leq n \\
&=\bigcup_{\sum n_{i} \leq n} \prod_{i \in \mathbb{N}} \mathscr{L}\left(Q_{n_{i}}\left(X_{i}\right)\right),
\end{aligned}
$$




$$
\begin{aligned}
\mathscr{L}\left(Q_{n}\left(\ell_{p}^{k}\left(X_{i}\right)\right)\right)=\left\{\prod_{i=1}^{k} T_{i}:\left(\prod_{i=1}^{k} T_{i}\right)\left(U_{\ell_{p}^{k}\left(E_{i}\right)}\right) \subset A_{n}\right\} \\
\quad \text { for some } A_{n} \in Q_{n}\left(\ell_{p}^{k}\left(X_{i}\right)\right) \\
\supset \frac{1}{k^{1 / p}} \prod_{i=1}^{k}\left\{T_{i}: T_{i} \in \mathscr{L}\left(Q_{n_{i}}\left(X_{i}\right), E_{i}, X_{i}\right), T_{i}\left(U_{E_{i}}\right) \subset A_{n_{i}}\right\} \\
\quad \text { for some } A_{n_{i}} \in Q_{n_{i}}\left(X_{i}\right), \sum n_{i} \leq n \\
=\frac{1}{k^{1 / p}} \bigcup_{\sum n_{i} \leq n} \prod_{i \in \mathbb{N}} \mathscr{L}\left(Q_{n_{i}}\left(X_{i}\right)\right) .
\end{aligned}
$$

THEOREM 2.18. Let $\left\{D_{i}\right\}_{i \in \mathbb{N}}$ be a sequence of bounded subsets of the Banach spaces $\left\{X_{i}\right\}_{i \in \mathbb{N}}$ with $D_{i} \subset X_{i}$, then for the bounded subsets $\ell_{\infty}\left(D_{i}\right)$ and $\ell_{p}^{k}\left(D_{i}\right)$ of the Banach spaces $\ell_{\infty}\left(X_{i}\right)$ and $\ell_{p}^{k}\left(X_{i}\right)$,

(1) $\sigma_{n}\left(\ell_{\infty}\left(D_{i}\right)\right)=\inf _{\sum n_{i} \leq n} \sup _{i \in \mathbb{N}} \sigma_{n_{i}}\left(D_{i}\right)$;

(2) $\inf _{\sum n_{i} \leq n} \sum_{i=1}^{k} \sigma_{n_{i}}\left(D_{i}\right) \leq \sigma_{n}\left(\ell_{p}^{k}\left(D_{i}\right)\right) \leq k^{1 / p} \inf _{\sum n_{i} \leq n} \sum_{i=1}^{k} \sigma_{n_{i}}\left(D_{i}\right)$.

Proof. (1) For the bounded subset $\ell_{\infty}\left(D_{i}\right)_{i \in \mathbb{N}}$ of the Banach space $\ell_{\infty}\left(X_{i}\right)_{i \in \mathbb{N}}$ and by using Lemma 2.16 , we get

$$
\begin{aligned}
\sigma_{n}\left(\ell_{\infty}\left(D_{i}\right)\right)_{i \in \mathbb{N}} & =\inf _{\prod_{i \in \mathbb{N}} T_{i} \in \mathscr{L}\left(Q_{n}\left(\ell_{\infty}\left(X_{i}\right)_{i \in \mathbb{N}}\right)\right)} \sup _{x \in \ell_{\infty}\left(D_{i}\right)} d\left(x, \prod_{i \in \mathbb{N}} T_{i}\left(U_{\left.\left.\ell_{\infty}\left(E_{i}\right)_{i \in \mathbb{N}}\right)\right)}\right)\right. \\
& =\sup _{\prod_{i \in \mathbb{N}} T_{i} \in \mathscr{L}\left(Q_{n}\left(\ell_{\infty}\left(X_{i}\right)_{i \in \mathbb{N}}\right)\right)} \inf _{x \in \ell_{\infty}\left(D_{i}\right)} d\left(x, \prod_{i \in \mathbb{N}} T_{i} \ell_{\infty}\left(U_{E_{i}}\right)\right) \\
& =\inf _{\sum n_{i} \leq n} \inf _{T_{i} \in \mathscr{L}\left(Q_{n_{i}}\left(X_{i}\right)\right)} \sup _{x_{i} \in D_{i}} \sup _{i \in \mathbb{N}} d\left(x_{i}, T_{i} U_{E_{i}}\right) \\
& =\inf _{\sum n_{i} \leq n} \sup _{i \in \mathbb{N}} \inf _{T_{i} \in \mathscr{L}\left(Q_{n_{i}}\left(X_{i}\right)\right)} \sup _{x_{i} \in D_{i}} d\left(x_{i}, T_{i} U_{E_{i}}\right) \\
& =\inf _{\sum n_{i} \leq n} \sup _{i \in \mathbb{N}} \sigma_{n_{i}}\left(D_{i}\right)_{i \in \mathbb{N}^{*}} .
\end{aligned}
$$

The proof of (2) is similar to (1).

COROLLARY 2.19. Let $\left\{R_{i}\right\}_{i \in \mathbb{N}}$ be a sequence of bounded linear operators with $R_{i} \in$ $L\left(E_{i}, X_{i}\right)$. For the bounded linear operators $\left(\oplus_{i \in \mathbb{N}} R_{i}\right) \in L\left(\ell_{\infty}\left(E_{i}\right)_{i \in \mathbb{N}}, \ell_{\infty}\left(X_{i}\right)_{i \in \mathbb{N}}\right)$ and $\left(\oplus_{i=1}^{k} R_{i}\right) \in L\left(\ell_{p}^{k}\left(E_{i}\right), \ell_{p}^{k}\left(X_{i}\right)\right)$,

(1) $\zeta_{n}\left(\oplus_{i \in \mathbb{N}} R_{i}\right)=\inf _{\sum n_{i} \leq n} \sup _{i \in \mathbb{N}} \zeta_{n_{i}}\left(R_{i}\right)$;

(2) $\inf _{\sum n_{i} \leq n} \sum_{i=1}^{k} \zeta_{n_{i}}\left(R_{i}\right) \leq \zeta_{n}\left(\oplus_{i=1}^{k} R_{i}\right) \leq k^{1 / p} \inf _{\sum n_{i} \leq n} \sum_{i=1}^{k} \zeta_{n_{i}}\left(R_{i}\right)$

Proof. (1) Let for every $i \in \mathbb{N}$, the subset $M_{i}=R_{i} U_{E_{i}}$ be the image of the unit ball of the Banach space $E_{i}$ under the operator $R_{i}$. Then from Definition 2.13 and by using Lemma 2.16 and Theorem 2.18, we get

$$
\begin{aligned}
\zeta_{n}\left(\oplus_{i \in \mathbb{N}} R_{i}\right) & =\sigma_{n}\left(\oplus_{i \in \mathbb{N}} R_{i}\left(U_{\ell_{\infty}\left(E_{i}\right)_{i \in \mathbb{N}}}\right)\right) \\
& =\sigma_{n}\left(\oplus_{i \in \mathbb{N}} R_{i}\left(U_{\ell_{\infty}\left(E_{i}\right)}\right)\right) \\
& =\inf _{\sum n_{i} \leq n} \sup _{i \in \mathbb{N}} \sigma_{n_{i}}\left(R_{i} U_{E_{i}}\right)_{i \in \mathbb{N}} \\
& =\inf _{\sum n_{i} \leq n} \sup _{i \in \mathbb{N}} \zeta_{n_{i}}\left(R_{i}\right)_{i \in \mathbb{N}},
\end{aligned}
$$




$$
\begin{aligned}
\zeta_{n}\left(\oplus_{i=1}^{k} R_{i}\right) & =\sigma_{n}\left(\oplus_{i=1}^{k} R_{i}\left(U_{\ell_{p}^{k}\left(E_{i}\right)}\right)\right) \\
& \leq \sigma_{n}\left(\oplus_{i=1}^{k} R_{i} \ell_{p}^{k}\left(U_{E_{i}}\right)\right) \\
& \leq k^{1 / p} \inf _{\sum n_{i} \leq n} \sum_{i=1}^{k} \sigma_{n_{i}}^{k}\left(R_{i} U_{E_{i}}\right) \\
& =k^{1 / p} \inf _{\sum n_{i} \leq n} \sum_{i=1}^{k} \zeta_{n_{i}}\left(R_{i}\right) .
\end{aligned}
$$

Similarly, we can prove that

$$
\inf _{\sum n_{i} \leq n} \sum_{i=1}^{k} \zeta_{n_{i}}\left(R_{i}\right) \leq \zeta_{n}\left(\oplus_{i=1}^{k} R_{i}\right) .
$$

\section{REFERENCES}

[1] A. Aksoy, Approximation schemes, related s-numbers and applications, thesis, University of Michigan, 1984.

[2] A. Aksoy and M. Nakamura, The approximation numbers $\gamma_{n}(T)$ and Q-precompactness, Math. Japon. 31 (1986), no. 6, 827-840.

[3] A. Pietsch, Operator Ideals, North-Holland Mathematical Library, vol. 20, North-Holland Publishing, Amsterdam, 1980.

[4] _ Approximation spaces, J. Approx. Theory 32 (1981), no. 2, 115-134.

Nashat Faried: Faculty of SCIENCE, Ain Shams University, Cairo, EgyPT

E-mail address: nfari ed@hotmai 1 . com

Zeinab Abd El-Kader: Faculty of Science, HelWan University, Cairo, Egypt

E-mail address: zeinab54@hotmai 1.com 


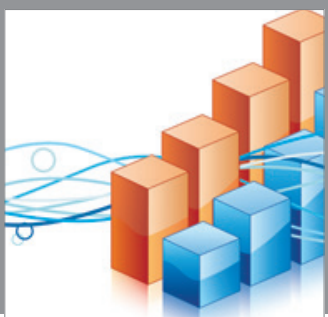

Advances in

Operations Research

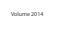

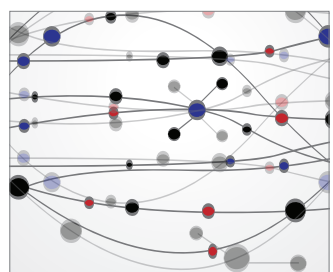

\section{The Scientific} World Journal
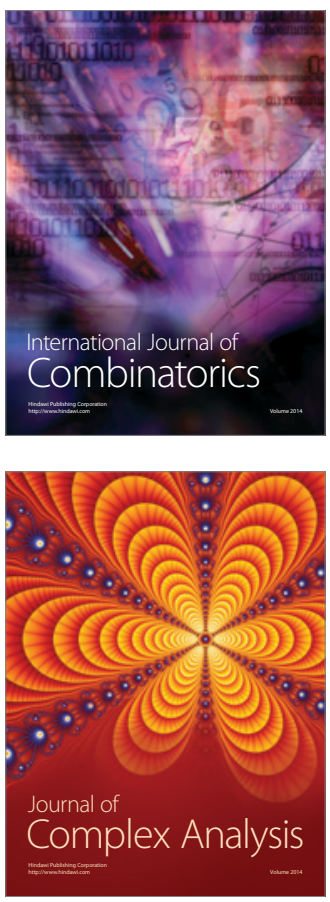

International Journal of

Mathematics and

Mathematical

Sciences
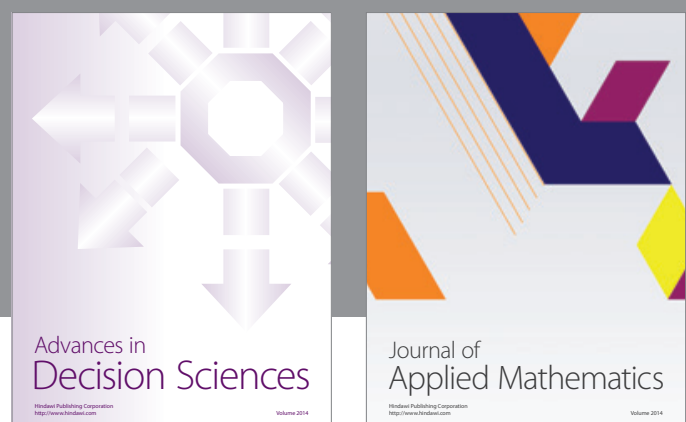

Journal of

Applied Mathematics
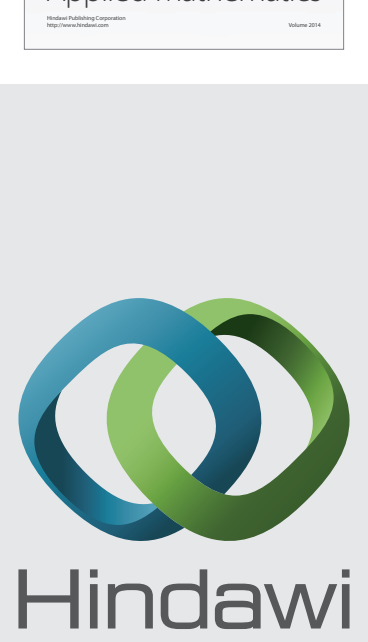

Submit your manuscripts at http://www.hindawi.com
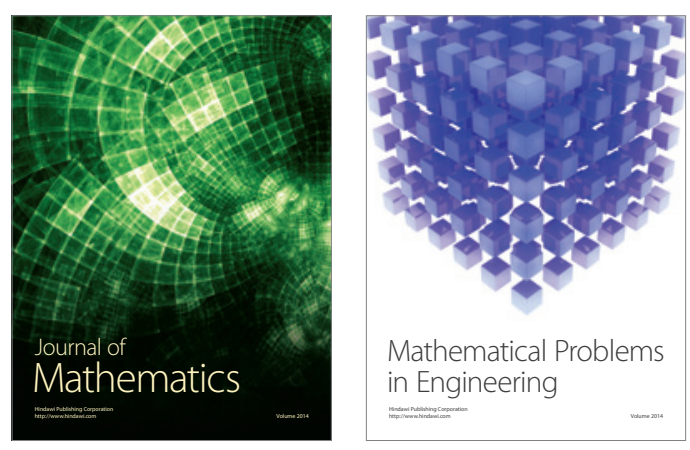

Mathematical Problems in Engineering
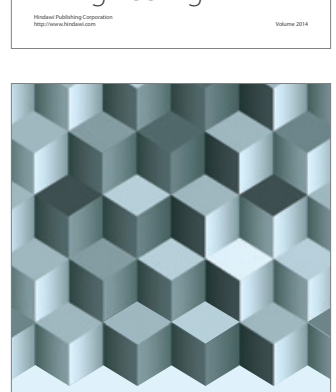

Journal of

Function Spaces
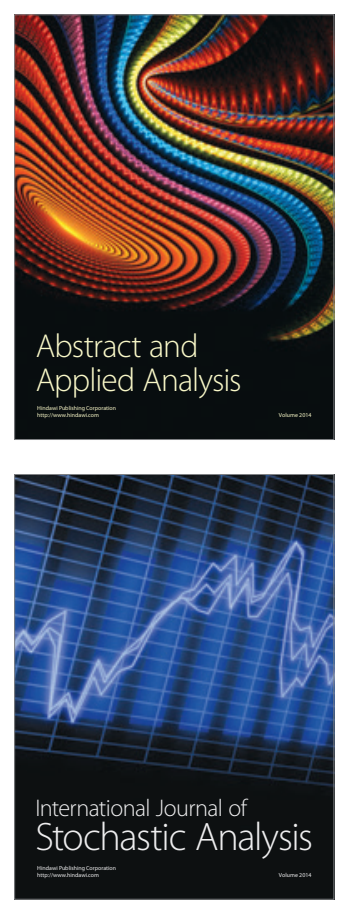

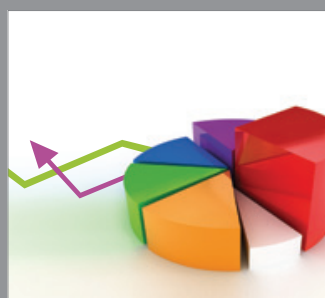

ournal of

Probability and Statistics

Promensencen
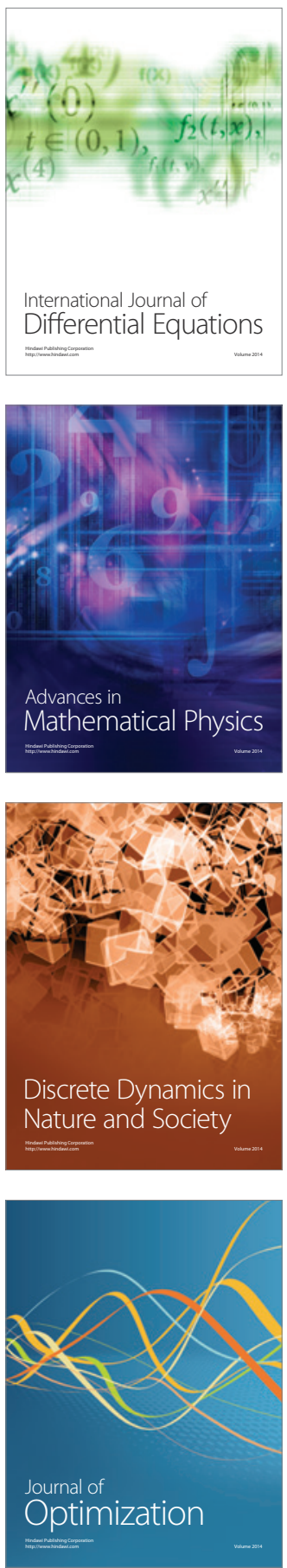